\title{
Motivos do abandono do seguimento médico no cuidado a portadores de hipertensão arterial: a perspectiva do sujeito
}

\author{
Reasons for medical follow-up dropout among patients \\ with arterial hypertension: the patient's perspective
}

M arli Teresinha Cassamassimo Duarte ${ }^{1}$

Antonio Pithon Cyrino ${ }^{2}$

Ana Teresa de Abreu Ramos Cerqueira ${ }^{3}$

$M$ aria Ines Battistella N emes ${ }^{4}$

M assako Iyda ${ }^{2}$

\footnotetext{
${ }^{1}$ Departamento de Enfermagem, Faculdade de M edicina de Botucatu, Universidade Estadual Paulista. Distrito de Rubião Júnior s/n, Rubião Júnior. 18618-970 Botucatu SP. mtduarte@fmb.unesp.br ${ }^{2}$ Departamento deSaúde Pública, Faculdade de M edicina de Botucatu, Universidade Estadual Paulista.

${ }^{3}$ Departamento de Neurologia, Psicologia e Psiquiatria, Faculdade de M edicina de Botucatu, UniversidadeEstadual Paulista.

${ }^{4}$ Departamento deM edicina Preventiva, Faculdade de M edicina, Universidade de São Paulo.
}

Abstract Arterial hypertension is a relevant public health problem as it is a risk factor for cardiovascular diseases, the major cause of mortality in Brazil. Low compliance and treatment dropout are among the main obstacles to individual control strategies. This study aimed at assessing the reasons for failure in medical appointment follow-up among a cohort of patients under treatment for four years in a primary care service. Thus, semi-structured interviews were conducted with 50 individuals who had dropped out of medical appointments. Statements were assessed by using thematic content analysis. The reported reasons for dropping out were most frequently related with the healthcare service itself - its organization and structure, and doctor-patient relationship - or treatment at another healthcare service. The reasons related with the patients themselves, such as absence of symptoms, improvement and/or normalization of arterial hypertension, as well as alcohol consumption contributed to the discontinuation of the treatment. To study the reasons for treatment dropout through the patients' eyes showed the abundance and diversity of problems involved.

Key words Arterial hypertension, D ropout, Primary healthcare, $\mathrm{H}$ ealthcare service
Resumo A hipertensão arterial é um expressivo problema de saúde pública enquanto fator de risco para as doenças cardiovasculares e principal grupo decausas demortalidadeno Brasil. A baixa adesão eo abandono do tratamento estão entreos principais obstáculos às estratégias individuais de controle. Estudam-se os motivos do abandono do seguimento médico em uma coorte de pacientes em tratamento de hipertensão arterial, em serviço de atenção primária à saúde, acompanhados por um período dequatro anos. Foram realizadas entrevistas semiestruturadas com cinquenta pessoas com hipertensão que abandonaram o seguimento médico. As respostas foram analisadas me diante a técnica de análise temática de conteúdo. Os motivos relatados para o abandono do seguimento mostraram predomínio de razões ligadas ao próprio serviço de saúde - sua organização, estrutura e a relação médico-paciente - e, ainda, tratamento em outro serviço de saúde. Razões de natureza psicossocial, como a ausência de sintomas, a melhora e/ou a normalização da pressão arterial e o consumo de álcool também contribuíram para 0 abandono do cuidado. Estudar os motivos do abandono na perspectiva do próprio sujeito permitiu verificar a riqueza e diversidade de problemas envolvidos no cuidado requerido. Palavras-chave Hipertensão arterial, Abandono, A tenção primária à saúde, Serviços de saúde 
Introdução

A elevação da pressão arterial é fator de risco independente, linear econtínuo para doença cardiovascular ${ }^{1}$ e 0 mais fortemente associado às doenças cerebrovasculares².

Inquéritos de base populacional ${ }^{3}$ realizados em algumas cidades do Brasil mostram prevalência de hipertensão arterial (pressão arterial $\geq$ $140 / 90 \mathrm{mmH} \mathrm{g}$ ) variando de $22,3 \%$ a $43,9 \%$.

A hipertensão arterial (HA) é, direta ou indiretamente, responsável pela maioria das complicações cardiovasculares, acarretando grande ônus à sociedade, seja por hospitalizações, invalidez ou mortes precoces ${ }^{4}$. No Brasil, em 2005, ocorreram mais de um bilhão deinternações por doenças cardiovasculares, com custo global de $R \$ 1.323 .775 .008,28^{3}$ e, em 2003, 27,4\% dos óbitos foram decorrentes de doenças cardiovasculares, atingindo $37 \%$, quando excluídos óbitos por causas mal definidas e por violência ${ }^{5}$. A elevada prevalência, a morbidade e mortalidade associadas e os custos sociais da hipertensão arterial constituem importantes problemas de saúde pública, cujo controle figura entre as prioridades, propostas pelo M inistério da Saúde, no Pacto pela Saúde- 2006 ena Política Nacional da Atenção Básica ${ }^{6}$.

Estudos ${ }^{7,8}$ realizados desde a década de sessenta têm demonstrado a eficácia da medicação anti-hipertensiva no controle da pressão arterial eredução da morbimortalidade associada. 0 tratamento medicamentoso tem indicações precisas; porém, a adoção de práticas que reduzam o consumo de sódio, o sedentarismo e a obesidade, entre outras medidas, estão indicadas a todos os pacientes ${ }^{3,9}$.

Inúmeros estudos, entretanto, têm apontado a enormedificuldade vivida pelas pessoas com hipertensão para persistirem seguindo as recomendações médicas, com expressiva frequência de abandono do tratamento ${ }^{10-15}$. Estima-se que cerca de dois terços dos pacientes em seguimento de HA não têm seus níveis pressóricos adequados devido, em grande parte, ao seguimento incorreto do tratamento medicamentoso ${ }^{3}$. Estudos internacionai $s^{10,11,16}$ e nacionais $s^{14,17-20}$ mostram grande variação nas taxas de adesão e de abandono observadas. Essa variação deve-se a vários fatores, entre eles o método de medida utilizado, o ponto de corte adotado para a definição de adesão e a seleç̧ão da amostra estudada.

A adesão ao seguimento, definida como 0 comparecimento às consultas médicas, num dado serviço de saúde, tem sido utilizada em vários estudos internacionais $s^{10,11}$ e nacionais ${ }^{18,19}$ como medida da adesão do paciente ao tratamento da HA. Embora se aceite que parte dos que abandonam o seguimento podem ter optado pelo seguimento em outros serviços, poucos trabal hos têm procurado investigar o problema ${ }^{21,22}$. Por outro lado, se reconhece que a assistência a pessoas com doenças crônicas, como a HA, o diabetes e outras, requer considerar a complexidade do cuidado (e do autocuidado) em condições de cronicidade. D estaca-se, nestereconhecimento, a crítica à abordagem estritamente técnica da adesão do paciente eà restrita consideração das dificuldades vividas em seu cotidiano ${ }^{23}$.

Reconhecendo a importância de melhor compreender esses aspectos das práticas de saúde, este estudo estabeleceu como objetivos identificar as razões expostas por portadores de HA para o abandono do seguimento médico em serviço de atenção primária à saúde e as estratégias que empregam para o controle da hipertensão arterial.

\section{Métodos}

Este artigo apresenta parte dos resultados da pesquisa "Estudo da assistência prestada aos portadores de hipertensão arterial em serviço de atenção primária à saúde" ${ }^{24}$, cujos sujeitos compõem uma coorte que incluiu o universo de usuários, do Centro de Saúde Escola da Faculdade de Medicina de Botucatu, com diagnóstico de hipertensão realizado em 1995. Os 192 pacientes quecompuseram essa coorte foram acompanhados num período compreendido entre 01/01/1995 e31/07/ 1999. 0 tempo deseguimento da coortevariou de três anos e meio (pacientes com diagnóstico em dezembro de 1995) a quatro anos emeio (pacientes diagnosticados em janeiro de 1995).

Considerou-se como abandono do seguimento o não comparecimento à consulta médica após 180 dias da data de agendamento da mesma. Os pacientes foram classificados em três grupos - aderente, abandono/aderente e abandono -, compostos, respectivamente, por 62 indivíduos que nunca abandonaram o seguimento médico, 41 com seguimento médico irregular e 89 que haviam abandonado o seguimento médico.

Os dados foram obtidos por meio de entrevista semiestruturada orientada por roteiro previamente testado. Dos 89 sujeitos em abandono de seguimento, entrevistaram-se cinquenta $(56,1 \%)$, aos quais se referem os resultados apresentados nesteartigo. Trinta enove (43,82\%) não 
foram entrevistados pelas seguintes razões: não localização (32), recusa (três), falta de condições físicas (dois) e óbito (dois).

As entrevistas foram realizadas pela primeira autora, de fevereiro a março de 2000, e ocorreram, em sua grande maioria, no domicílio do entrevistado. As informações foram anotadas à medida que iam sendo prestadas, procurandose registrá-las literalmente.

A análise temática de conteúdo foi a técnica utilizada para o tratamento das respostas dos entrevistados ${ }^{25}$. Esta modalidade de análise permite, segundo Blanchet e Gotman ${ }^{26}$, um recorte transversal do corpus, tendo o tema como unidade, desfazendo-se a singularidade do discurso, e recortando-se o que, de uma entrevista a outra, se referir ao mesmo tema.

O projeto dessa pesquisa foi aprovado pela Comissão de Ética em Pesquisa da Faculdade de M edicina de Botucatu e todos os entrevistados assinaram termo de consentimento livre e esclarecido.

\section{Resultados}

\section{Caracterização dos sujeitos}

A maioria (62\%) dos entrevistados era do sexo feminino, predominando os pacientes com sessenta anos ou mais (28\%), seguidos dos que tinham idade entre quarenta e 49 anos (26\%), sendo que apenas três pacientes (6\%) tinham menos de trinta anos. M ais de dois terços (78\%) eram casados. 0 nível de escolaridade predominante foi o primeiro grau incompleto (64\%), embora 24\% tivessem o segundo grau completo ou mais anos de estudo. Com relação à posição na ocupação, o percentual de entrevistados que trabal havam, $52 \%$, foi discretamente maior que o dos que não trabal havam (48\%).

Quanto à ocupação, observou-seinserção em vários setores produtivos, sendo que a maioria, $58 \%$, atuava na área de prestação de serviços; $15 \%$ trabalhavam no comércio; $15 \%$, em áreas sociais como saúde e educação, e 11\%, na agricultura.

O material empírico foi analisado buscandose identificar e recortar referências aos motivos para 0 abandono do seguimento médico e estratégias utilizadas pelos portadores no controle da hipertensão.
Motivos de abandono

do seguimento médico

0 abandono do seguimento médico, no serviço de saúde em estudo, não significou, efetivamente, abandono de cuidado e, até mesmo, de seguimento, uma vez que $56 \%$ (28) dos entrevistados estavam frequentando outros serviços de saúde para o tratamento da HA.

Dentre as causas relatadas para 0 abandono do seguimento médico no serviço investigado, predominaram as razões ligadas à própria instituição - sua organização e estrutura e características da relação médico-paciente -, seguidas daquelas denominadas psicossociais, e, ainda, as relacionadas à busca de tratamento em outro serviço de saúde, à ausência de sintomas, à normalização da pressão arterial (PA) e à distância do domicílio ao serviço.

A organização e estrutura do serviço enquanto causas do abandono foram decorrência de dificuldades, incompatibilidades einsatisfações com diferentes dimensões dos serviços prestados pela instituição, dentre as quais se destacaram o intervalo longo entre as consultas; dificuldade para agendar consulta; demora em ser atendido; horário de atendimento incompatível com o do trabalho e/ou com as ocupações diárias; mudança de médico responsável pela assistência e impossibilidade de acesso ao médico especialista. Alguns entrevistados relataram terem buscado tratamento em outros serviços de saúde motivados por esses fatores: Lá, na ocasião, estava com falta de médico. Num ano só me consultaram uma vez. Aí eu não fui mais e, também, porque demorava muito para ser atendida. Pedi pra minha filha me colocar no convênio [de saúde]. (M aria de Fátima, que já frequentava outro serviço de saúde)

Eu já procurei pra marcar, mas ainda não estavam marcando. É difícil pra marcar consulta. (Lucinda, sem seguimento médico)

A relação médico-paciente foi outro motivo relatado pelos sujeitos como causa do abandono do seguimento no serviço, expresso como segue: descontentamento, constrangimento ou insatisfações, como fatores apontados para o abandono do seguimento na unidade, que, em al guns casos, motivaram, também, a procura por outro serviço de saúde: Pela maneira como fui tratada dentro do consultório, da maneira como conversou, da maneira como expôs meu corpo. [0 médico me] falou:"tem mania decomer igual porCo". 0 gordo não gosta de ser tratado de gordo. Perdi todo pique de continuar. (M iriam, frequentava outro serviço de saúde) 
Porque ninguém se interessou pela minha dor decabeça. $\mathrm{N}$ ão mesenti bem atendida pelo médico. (Antonia, frequentava outro serviço de saúde)

Para outros entrevistados, o abandono do seguimento no serviço decorreu da saída de profissional com o qual tinha confiança e boa relação. Alguns deles passaram a ser atendidos pelo mesmo médico em outra instituição de saúde: Porque meu médico saiu de lá. Mudou-se. Como elejá trabalhava no hospital, eu passei a ser atendida lá, por ele. (Elvira, frequentava outro serviço de saúde)

As razões aqui denominadas de psicossociais compreenderam motivos de caráter mais subjetivos (como timidez, medo, falta de motivação e abuso de álcool) e objetivos (dificuldades financeiras, ligadas ao trabalho eà família, limitações físicas, baixa escolaridade e mudança de cidade ou bai rro). Esses foram reconhecidos tanto pelo grupo de pacientes que frequentava outro serviço de saúde, como também pelo grupo sem seguimento médico: Às vezes, não tenho dinheiro pra ir no dia da consulta e, também, porqueeu não sei ler, às vezes, passa o dia da consulta. (A parecida, que frequentava outro serviço de saúde)

Porque minha filha trabalha no hospital e me leva lá. É mais fácil pra ela me levar. (Alina, frequentava outro serviço de saúde)

Os relatos de Alina e de outros entrevistados expressaram condição bastante comum entre os idosos, queéa dependência de um acompanhante ou cuidador, limitando a sua autonomia para frequentarem um serviço de saúde de sua escolha.

A ausência ou remissão de sintomas, bem como a normalização da pressão arterial, foram relatadas como causas para o abandono do seguimento médico: Na minha opinião, eu não via necessidade de vir, porque nunca senti dores. Eu achei que tava bom. (Antonio Carlos, frequentava outro serviço de saúde)

Algumas vezes atéexpondo a ausência deuma noção de direito à assistência: Porque eu não sinto nada. Graças a Deus, tô bom. E a gente tando bom, tem que deixar o lugar pra quem precisa. (Carmelino, sem seguimento médico)

A ausência de melhora com o tratamento foi também apontada como razão para 0 abandono do seguimento médico: Porque todas as consultas que tirava lá era a mesma coisa. Não tinha novidade. Não via melhora. A música era quase todos os dias iguais. A gente quando vê que toma remédio e não melhora, a gente precisa ter esperança. (Francisca, sem seguimento médico)

A procura por tratamento em outro serviço de saúde foi apresentada como causa de aban- dono e relacionada pelos entrevistados ao acesso a serviços privados por convênio desaúde; à busca por serviço mais próximo da residência e ao tratamento em unidade especializada, associando essas causas a uma crítica ao serviço estudado: Porque minha filha fez um convênio [de saúde] e lá é mais rápido. Os exames são feitos na hora. Numa ocasião que teve greve, eu não pude fazer os exames e fiquei mais de um ano sem ser atendida. la e não dava certo. Aí veio o convênio que facilitou. (Dirce, frequentava outro serviço de saúde)

Para alguns entrevistados, o consumo abusivo do álcool foi o motivo do abandono do seguimento médico: Todas as vezes que comecei tratamento pra pressão, eu parei, por causa da bebida. (Aparecido, sem seguimento médico)

\section{Estratégias empregadas \\ para controle da hipertensão arterial}

A mai oria dos participantes dessa pesquisa, a despei to do abandono do seguimento no serviço estudado, empregava diferentes estratégias para o controle da PA, dentre as quais o uso da medicação anti-hipertensiva; realização de atividade física; adoção da recomendação dietética; verificação regular da pressão arterial; uso de terapias casei ras e seguimento médico em outros serviços de saúde.

Aqueles que relataram utilizar a medicação prescrita o fizeram de modo regular, irregular e esporádico. 0 uso regular da medicação antihipertensiva foi relatado por $45 \%$ (dezenove) dos entrevistados, assim caracterizados por utilizarem-na de modo regular (diária e ininterrupta) no momento da entrevista. A maioria desses (14\%) referiu nunca ter interrompido o uso da medicação, apresentando expressões como "nunca parei", "é sagrado", "é religião pra mim".

O uso irregular da medicação foi identificado em $14 \%$ (sete) dos entrevistados com base em relatos de não tomada de uma das doses em um dia, interrupção por alguns dias ou abandono de uso de parte da medicação prescrita.

A utilização da medicação anti-hipertensiva era feita por $21 \%$ (nove) dos sujeitos, apenas quando havia a presença de sintomas ou elevação da pressão arterial, assim manifestada: "tomo o remédio de vez em quando", "tomo quando acho que a pressão sobe", "tomo quando sinto alguma coisa".

O seguimento da dieta prescrita foi relatado por $28 \%$ (catorze) dos entrevistados, enquanto $60 \%$ (trinta) delesinformaram quefaziam alguma restrição no consumo desal de cozinha. As associ- 
ações de várias recomendações demedidas decontrole foram relatadas, sendo as mais frequentes a restrição de sal e dieta alimentar; restrição de sal, dieta alimentar erealização deatividadefísica erestrição de sal e realização de atividade física.

M ais da metade (56\%) dos entrevistados relatou utilizar outros serviços de saúde para tratamento da HA, destacando-se, entre esses, unidades de natureza ambulatoriais públicas e privadas. Apenas uma pessoa referiu frequentar pronto-socorro público.

A verificação, regular ou esporádica, da pressão arterial como medida de controle foi relatada por $72 \%$ (36) dos entrevistados. Terapias caseiras mencionadas por alguns entrevistados foram a infusão de folhas de chuchu e dentes de alho em água.

\section{Discussão}

Os dados aqui obtidos, bem como os relatados por Oignam et al. ${ }^{17}$, sugerem que a adesão ao seguimento médico, num dado serviço, não é expressão final da assistência utilizada pelo paciente, uma vez que o mesmo pode buscar outros serviços, o que, também, se aplica ao tratamento com um todo.

Relatos apontando a organização e estrutura de funcionamento do serviço de saúde como motivos do abandono do seguimento médico na instituição denotaram a percepção dos pacientes das diferentes barreiras impostas pelo serviço, especialmente aquelas que restringiam 0 acesso ao cuidado. Dentre tais obstáculos, apontaram a mudança frequente de médico como uma das razões para o abandono do seguimento, o que limitou a continuidade do cuidado médico - condição essencial para o estabelecimento de vínculo e confiança -, e, consequentemente, de uma boa relação médico-paciente. Considerando que a maioria dos entrevistados trabal hava, pode-se compreender por que o horário defuncionamento da unidade de saúde, bem como o tempo de espera para ser atendido, foram apontados como motivos para o abandono do seguimento.

Diante de dificuldades impostas pela organização do serviço ou de sua relação com o médiCo, os pacientes assumiram comportamentos diferentes quanto a sua motivação para 0 tratamento. Assim, al guns buscaram atendimento em outro serviço de saúde, enquanto outros ficaram sem acompanhamento médico.

A influência da estrutura e organização do serviço de saúde e a qualidade da relação médi- co-paciente na adesão foram bem estabelecidas em vários estudos sobre doenças crônicas em geral, especialmente enfocando os aspectos aqui relatados ${ }^{27-29}$.

A realização de tratamento em outro serviço, enquanto motivo de abandono, permitiu reconhecer distintas restrições de acesso ao serviço de saúde, aquelas de natureza geo gráfica e as relativas à própria organização da instituição.

Como já descrito, alguns pacientes afirmaram que abandonaram o seguimento no serviço estudado por terem acesso a serviços privados, financiados por seu convênio de saúde, ou a serviços de aten ção secundária. N os relatos de frequência aos serviços conveniados, observou-sea expressão de uma série de vantagens dessas organizações, o que contrasta com as barreiras já apontadas. Os relatos que expressaram a busca de tratamento de outra condição, em serviço de atenção secundária, como motivo do abandono do seguimento, não apontaram a percepção de barreiras no atendimento do serviço estudado e, sim, maior facilidade de tratamento num único serviço de saúde.

A distância entre a moradia e o serviço foi claramente relatada como um obstáculo ao seguimento. Cohn et al. ${ }^{30}$ referem que a proximidade do serviço de saúde, entre outros fatores, rege a orientação de seguimentos sociais urbanos periféricos, para a utilização de serviços de saúde.

Os motivos de abandono do seguimento médico de natureza psicossocial foram classificados, segundo Di M atteo eDi Nicolla ${ }^{27}$, em subjetivos (fatores intrapsíquicos envolvendo pensamentos, sentimentos eatitudes) eobjetivos (fatores ambientais correspondendo a aspectos de vida dos pacientes). Vários autores ${ }^{27,29}$ reconhecem que as crenças e normas subjetivas que os pacientes constroem emantêm influenciam, substancialmente, na sua adesão.

Outros estudos ${ }^{11,31}$ encontraram relatos de causas semelhantes, em sua maioria, às aqui relatadas. Caldwell et al. ${ }^{11}$ observaram, além dessas, a falta de apoio familiar, e Florenzano et al. ${ }^{31}$ relataram número excessivo de comprimidos prescritos e duração do tratamento. N enhuma dessas pesquisas encontrou, como motivo de abandono deseguimento, o consumo deálcool e o tratamento em outros serviços de saúde, aqui relatados.

O abuso do álcool apontado como razão para o abandono do seguimento, na unidade de saúde investigada, bem como em outros serviços de saúde, já foi bem documentado nos tra- 
balhos sobre abandono do tratamento de tuberculose $^{32} \mathrm{e}$ a não adesão ao tratamento da aids ${ }^{33}$.

Em relação às estratégias utilizadas para controle da hipertensão arterial, observou-sequea maioria dos pacientes entrevistados rel atou que empregava uma ou mais ações para controlar a HA.

A medicação anti-hipertensiva, ainda queutilizada de forma irregular por alguns pacientes, ou mesmo de forma esporádica por outros, foi uma das ações bastante relatadas para controle da $\mathrm{HA}$, como verificado em outra investigação ${ }^{17}$.

Nesse mesmo sentido, Martins ${ }^{34}$ afirma que a medicação anti-hipertensiva passa a fazer parte obrigatória do cotidiano do paciente, mesmo que as instruções médicas não sejam seguidas à risca, como no caso daqueles que a usam irregularmente ou apenas na presença de sintomas.

Pesquisa ${ }^{17}$ realizada em serviço ambulatorial público, questionando pacientes que também foram previamente classificados como em abandono do seguimento médico, observou que $60 \%$ dos sujeitos referiram utilizar regularmente a medicação e 10\% faziam uso irregular.

Uma das estratégias de controleutilizada pelos sujeitos deste estudo foi a verificação da pressão arterial, como também observado em outra investigação ${ }^{12}$, na qual se identificou o automanejo dos medicamentos em função da sintomatologia percebida, deixando deutilizá-lo e/ou decumprir com as recomendações e o seguimento médico.

Tal aspecto é particularmente importante, uma vez que os pacientes mantêm a regularidade do tratamento medicamentoso e, até mesmo, o seguimento ou não das recomendações para mudanças no estilo de vida e do acompanhamento médico, recorrendo à percepção de sintomas ou do nível da pressão arterial. As autoavaliações do nível pressórico como adequado ou normal podem ser resultado do tratamento, que, quando interpretadas como cura, provocam a interrupção da medicação, possivelmente acarretando, em pouco tempo, o descontroleda pressão arterial.

M ais da metade (56\%) das pessoas entrevistadas relatou que estava sen do acompanhada em outros serviços de saúde para o tratamento da
HA. Dados semelhantes foram relatados por Oigman et al. ${ }^{17}$ que, com base em registros dos prontuários médicos, obtiveram uma taxa de $70 \%$ de abandono do seguimento médico, no ambulatório de HA. Porém, ao entrevistarem os pacientesinicialmente considerados em abandono, observaram que praticamente a metade relatou estar em acompanhamento em outros serviços de saúde.

A penas cerca de um terço dos pacientes relatou mudanças no estilo de vida como estratégia de controle da HA. A baixa adesão ao tratamento não medicamentoso vem confirmar os dados da literatura que apontam menor adesão dos pacientes aos regimes de tratamento que requeiram realização permanente de mudanças de estilo de vida $29,35,36$.

\section{Conclusão}

0 problema do abandono do tratamento é um dos aspectos fundamentais no controle individual da hipertensão arterial, uma vez que pode comprometer sua efetividade, com consequências sérias para o próprio paciente, sua família e comunidade.

Constatou-se que o abandono do seguimento médico em serviço de saúde não corresponde ao abandono do tratamento, uma vez que parcela expressiva dos que deixaram de frequentar a instituição estudada mantinham parte das medidas prescritas e/ou frequentavam outros serviços.

Estudar os motivos do abandono na perspectiva do próprio sujeito permitiu verificar a riqueza e diversidade de problemas envolvidos no cuidado requerido. Os resultados alcançados mostram a necessidade de os serviços de saúde identificarem aspectos de sua organização que, ao restringirem 0 acesso dos usuários, limitam suas possibilidades de autocuidado. No âmbito da assistência, cabe aos profissionais orientarem suas práticas na identificação, conjunta com seus pacientes, dos diversos obstáculos presentes no cotidiano destes, bem como no apoio a seu enfrentamento. 


\section{Colaboradores}

M TC Duarte participou da revisão da literatura, elaboração do projeto, coleta de dados, análise dos resultados e redação final. AP Cyrino participou da revisão da literatura, el aboração do projeto, análise dos resultados e redação final. ATAR Cerqueira contribuiu para a orientação metodológica, análise dos resultados e redação final do artigo. M IB N emes colaborou com a elaboração do projeto, análise dos dados e revisão do artigo. M Iyda contribuiu com a orientação do projeto e análise dos resultados.

\section{Referências}

1. Levington S, Clarke R, Oizilbash N, Peto R, Collins $\mathrm{R}$, for the Prospective Studies Collaboration. Agespecific relevance of usual blood pressure to vascular mortality: a meta-analysis of individual data for one million adults in 61 prospective studies. Lancet 2002; 360:1903-1913.

2. Kannel WB, Wolf PA, MC Gree D, Dawber TR, M CN amara P, Castelli P. Systolic blood pressure, arterial rigidity and risk of stroke. The Framingham Study. J Am M ed Assoc 1981; 245(12):1225-1229.

3. Sociedade Brasileira de Cardiologia. Sociedade Brasileira de Hipertensão. Sociedade Brasileira de $\mathrm{Ne}$ frologia. V Diretrizes Brasileiras de Hipertensão Arterial. São Paulo: Sociedade Brasileira de Cardiologia. Sociedade Brasileira de Hipertensão. Sociedade Brasileira de N efrologia; 2006.

4. Lessa I. Estudos brasileiros sobre a epidemiologia da hipertensão arterial: análise crítica dos estudos de prevalência. Inf Epidemiol SUS 1993; 2:59-75.

5. Lotufo PA. Stroke in Brazil: a neglected disease. São Paulo Med J 2005; 123(1):3-4.

6. Brasil. M inistério da Saúde. Secretaria de Atenção Básica. Departamento de Atenção Básica. Política Nacional de Atenção Básica. Brasília: Ministério da Saúde; 2006.

7. Veterans Administration Cooperative Study Group on Antihypertensive Agents. Effects of treatment on morbidity in hypertensive II: results in patients with diastolic blood pressure averaging 90 through $114 \mathrm{mmH}$. J Am Med Assoc 1974; 213:1143.

8. Flack JM, Novikov SV, Ferrario CM. Benefits of adherence to anti-hypertensive drug therapy. Eur Hearth J 1996; 17(Sup A):16-20.

9. The Seventh Report of the Joint National Committee on Prevention, Detection, Evolution and Treatment of High Blood pressure. The JNC 7 report. J Am M ed Assoc 2003; 289(19):2560-2572.

10. Florenzano UR, Roessler E, Scharazer J, Soto I, Cantu AM. Permanencia en tratamiento antihipertensivo: comparación de los sistemas de seguimiento. Bol Oficina Sanit Panam 1981; 91(5):428-440.

11. Caldwell JR, Cobb S, Dowling MD, Jongh D. The dropout problem in antihypertensive treatment. A pilot study of social and emotional factors influencing a patient's ability to follow antihypertensive treatment. J Chron Dis 1970; 20:579-592.

12. Cade NV. Aspectos psicossociais no cotidiano dos hipertensos: um estudo sobre a continuidade do tratamento em pacientes pertencentes ao programa para controle da hipertensão arterial. Vitória: Universidade Federal do Espírito Santo; 1994.

13. Car MR. A mortalidade cárdio-cerebrovascular e os problemas da prática do controle da hipertensão arterial. Rev Esc Enf USP 1998; 32(2):140-143.

14. Busnello RG, M elchior R, Faccin $C$, Vettori D, Petter J, M oreira LB, Fuchs FD. Characteristics Associated with the dropout of hypertensive patients followed up in an outpatient referral clinic. Arq Bras Cardiol 2001; 76(10):352-354.

15. Souza LB, Souza RKT, Sccochi MJ. Hipertensão arterial e saúde da família: atenção aos portadores em município de pequeno porte na região Sul do Brasil. Arq Bras Cardiol 2006; 87(1):496-503. 
16. Hershey JC, Morton BG, Davis JB, Reichgott MJ Patient compliance with antihypertensive medication. Am J Public Health 1980; 70(10):1081-1088.

17. Oigman W, Cordeiro HA, Benchimol AB, Quadra AAF. Abandono de tratamento na hipertensão arterial. Arq Bras Cardiol 1977; 30(4):247-253.

18. Lessa I, Evangelista Filho D, Santo MEGE. Adesão, eficácia e custo do tratamento da hipertensão arterial. Arq Bras Cardiol 1983; 41(2):119-123.

19. Giorgi DMA, Mion Júnior $D, C$ ar $M R$, Pierin $A M G$, Silva $H B, M$ arcondes $M$. Aderência ao tratamento em hipertensão arterial: influência de variáveis estruturais e de estratégias que visem sua melhora. Rev Bras Med Cardiol 1985;4(4):167-176.

20. Rocha JMA. Compliance. Você acha que a receita vai ser seguida? In: Paulo LG, Zanini AC. Compliance: sobre o encontro paciente-médico. São Paulo: IPEX; 1997. p. 51-66.

21. Safran DG, M ontgomery JE, Chang H, Murphy J, Rogers WH. Switching doctors: predictors of voluntary disenrollment from a primary physician's practice. J Fam Pract 2001; 50(2):130-136.

22. Smith MA, Bartell JM. Changes in usual source of care and perceptions of health care access, quality, and use. Med Care 2004; 42(10):975-984.

23. Cyrino AP, Schraiber LB, Teixeira RR. Education for type 2 diabetes mellitus self-care: from compliance to empowerment. Interface (Botucatu) 2009; 13(30):93-106

24. Cyrino AP, Nemes MIB, Duarte MTC. Estudo da assistência prestada aos pacientes portadores de hipertensão arterial em serviço de atenção primária à saúde 1995 a 1998 [Projeto de pesquisa]. Botucatu; 1999.

25. Bardin L. Análise de conteúdo. Lisboa: Edições 70; 1997.

26. Blanchet A, Gotman A. A enquete e seus métodos: a entrevista. Paris: Editions Nathan; 1992

27. Di Matteo MR, Di Nicola DD. Achieving patient compliance: the psychology of the medical practitioner's role. New York: Pergamon Press; 1982.

28. Becker $\mathrm{MH}$. Patient adherence to prescribed therapies. Med Care 1985; 23:539-555

29. M eichenbaum $D$, Turk DC. Facilitating treatment adherence: a practitioner's guidebook. New York: Plenun Press; 1987.
30. Cohn A, Nunes E, Jacobi P, Karschu US. A medicalização e o imaginário no consumo de serviço de saúde. In: A saúde como direito e como serviço. São Paulo: Cortez; 1991. p. 95-130.

31. Florenzano UR, Scharager J, Contu AM, Soto I. Un estudio de pacientes que abandonam su tratamiento antihipertensivo. Rev M ed Chil 1981; 109:1060-1064.

32. M artínez GMC, Guiscafré GH, Fleitas E. E, Cedillo $\mathrm{HML}$, Muñoz-Cota M C, Muñoz HO. Fatores que influyen en el abandono del tratamiento antituberculoso y valoración de un programa de vigilancia familiar. Rev Enferm Inst M ex Seguro Soc 1982; 20(6):667-675.

33. Nemes MIB, M elchior R, Jordan MS, Okazaki E, Komatsu CL. Análise qualitativa de entrevistas semiestruturadas com usuários. In: Ministério da Saúde. Coordenação Nacional de DST e AIDS. Aderência ao tratamento por anti-retrovirais em serviços públicos no Estado de São Paulo. Braślia: M inistério da Saúde; 2000. p. 103-113.

34. Martins STF. Cotidiano e emoç̃es no processo saúdedoença: análise psicossocial da hipertensão arterial. São Paulo: Pontifícia Universidade Católica; 1994.

35. Gerber KE, Nehemkis AM. Compliance. The dilemma of the chronically ill. New York: Springer Publishing Company; 1986.

36. Pessuto J. M ecanismos de coping utilizados por indivíduos portadores de hipertensão arterial. Ribeirão Preto: Escola de Enfermagem, Universidade de São Paulo; 1999.

Artigo apresentado em 09/10/2007

Aprovado em 01/09/2008

Versão final apresentada em 30/10/2008 